\title{
The role of diffusion tensor imaging and tractography in the surgical management of brainstem gliomas
}

\author{
*Xiong Xiao, MD,' Lu Kong, MD, ${ }^{1}$ Changcun Pan, MD,1 Peng Zhang, MD," Xin Chen, MD,1 \\ Tao Sun, MD, ${ }^{1}$ Mingran Wang, MD, ${ }^{2,3}$ Hui Qiao, MD, ${ }^{2,3}$ Zhen Wu, MD, ${ }^{1}$ Junting Zhang, MD, ${ }^{1}$ and \\ Liwei Zhang, MD ${ }^{1,4}$ \\ Departments of ${ }^{1}$ Neurosurgery and ${ }^{3}$ Neuroelectrophysiology, Beijing Tiantan Hospital, Capital Medical University; ${ }^{2}$ Beijing \\ Neurosurgical Institute, Capital Medical University; and ${ }^{4}$ China National Clinical Research Center for Neurological Diseases \\ (NCRC-ND), Beijing, China
}

OBJECTIVE Diffusion tensor imaging (DTI) and diffusion tensor tractography (DTT) have the ability to noninvasively visualize changes in white matter tracts, as well as their relationships with lesions and other structures. DTI/DTT has been increasingly used to improve the safety and results of surgical treatment for lesions in eloquent areas, such as brainstem cavernous malformations. This study aimed to investigate the application value of DTI/DTT in brainstem glioma surgery and to validate the spatial accuracy of reconstructed corticospinal tracts (CSTs).

METHODS A retrospective analysis was performed on 54 patients with brainstem gliomas who had undergone surgery from January 2016 to December 2018 at Beijing Tiantan Hospital. All patients underwent preoperative DTI and tumor resection with the assistance of DTT-merged neuronavigation and electrophysiological monitoring. Preoperative conventional MRI and DTI data were collected, and the muscle strength and modified Rankin Scale (mRS) score before and after surgery were measured. The surgical plan was created with the assistance of DTI/DTT findings. The accuracy of DTI/DTT was validated by performing direct subcortical stimulation (DsCS) intraoperatively. Multiple linear regression was used to investigate the relationship between quantitative parameters of DTI/DTT (such as the CST score and tumorto-CST distance [TCD]) and postoperative muscle strength and mRS scores.

RESULTS Among the 54 patients, 6 had normal bilateral CSTs, 12 patients had unilateral CST impairments, and 36 had bilateral CSTs involved. The most common changes in the CSTs were deformation $(n=29)$, followed by deviation $(n=28)$ and interruption $(n=27)$. The surgical approach was changed in 18 cases $(33.3 \%)$ after accounting for the DTI/ DTT results. Among 55 CSTs on which DsCS was performed, 46 (83.6\%) were validated as spatially accurate by DsCS. The CST score and TCD were significantly correlated with postoperative muscle strength $(r=-0.395, p<0.001$, and $r$ $=0.275, p=0.004$, respectively) and postoperative mRS score $(r=0.430, p=0.001$, and $r=-0.329, p=0.015$, respectively). The CST score was independently linearly associated with postoperative muscle strength $(t=-2.461, p=0.016)$ and the postoperative mRS score $(t=2.052, p=0.046)$.

CONCLUSIONS DTI/DTT is a valuable tool in the surgical management of brainstem gliomas. With good accuracy, it can help optimize surgical planning, guide tumor resection, and predict the postoperative muscle strength and postoperative quality of life of patients.

https://thejns.org/doi/abs/10.3171/2020.10.FOCUS20166

KEYWORDS diffusion tensor imaging; brainstem gliomas; surgical management

ABBREVIATIONS BTH-CMU = Beijing Tiantan Hospital, Capital Medical University; CST = corticospinal tract; DFOV = display field of view; DsCS = direct subcortical stimulation; DTI = diffusion tensor imaging; DTT = diffusion tensor tractography; MEP = motor evoked potential; ML = medial lemniscus; $\mathrm{mRS}$ = modified Rankin Scale; ROI $=$ region of interest; $T C D=$ tumor-to-CST distance.

SUBMITTED February 28, 2020. ACCEPTED October 23, 2020

INCLUDE WHEN CITING DOI: 10.3171/2020.10.FOCUS20166.

${ }^{*}$ X.X. and L.K. contributed equally to this work. 
B RAINSTEM gliomas comprise a type of glioma that originates from the midbrain, pons, and medulla, accounting for $10 \%-20 \%$ of all intracranial tumors in children and $2 \%-4 \%$ of all intracranial tumors in adults. ${ }^{1-3}$ Brainstem gliomas can be divided into four types based on MRI characteristics: diffuse, focal, exophytic, and cervicomedullary junction tumors. ${ }^{4}$ Surgery has been reported to be beneficial for all of these types, except diffuse tumors. ${ }^{5-7}$ However, the high density of neural structures and critical neurological function make brainstem glioma surgeries extremely challenging.

In recent years, multimodal techniques-including functional MR, neuronavigation, intraoperative MRI, and intraoperative electrophysiological monitoring-have been increasingly used in clinical practice to improve the safety and efficacy of resections. Among these techniques, diffusion tensor imaging (DTI) and diffusion tensor tractography (DTT) have the ability to noninvasively visualize white matter tracts as well as their correlation with lesions and other structures. ${ }^{8}$ Additionally, DTI has been reported to be helpful in both the surgical planning and prediction of postoperative neurological function..$^{9-13}$ However, most of these applications are confined to supratentorial gliomas in eloquent areas and, most recently, brainstem cavernous malformations. The utility of DTI/DTT in brainstem glioma surgery has rarely been discussed. In addition, controversy persists regarding the spatial accuracy of DTI/DTT.

In this study, we primarily aimed to investigate the application value of DTI/DTT in brainstem glioma surgery, specifically the influence on the selection of surgical approaches and the relationship between DTI/DTT findings and postoperative muscle strength, as well as quality of life. In addition, the spatial accuracy of DTI/DTT on corticospinal tracts (CSTs) has been validated based on intraoperative neuronavigation-guided direct subcortical stimulation (DsCS).

\section{Methods \\ Study Population}

A retrospective review identified a cohort of consecutive patients with brainstem gliomas who underwent surgery at Beijing Tiantan Hospital, Capital Medical University (BTH-CMU) from January 2016 to December 2018. The inclusion criteria were as follows: 1) patients with pathologically confirmed brainstem glioma; 2 ) patients with focal or exophytic tumors; 3) patients whose surgical plans were made with the assistance of DTI/DTT findings; and 4) patients who underwent surgery with the aid of multimodal image-merged neuronavigation. The exclusion criteria were as follows: 1) patients with incomplete records of clinical or radiological data who could not meet the study requirements; 2) patients who expressed an unwillingness to be included in the studies; and 3) patients whose surgery was performed as a biopsy procedure other than tumor resection. The study was approved and informed consent from patients waived by the IRB of BTH-CMU.

Demographic, clinical, radiological, pathological, and surgical data were collected and double-checked by two neurosurgeons. In addition, the modified Rankin Scale
(mRS) score and muscle strength were assessed at both admission and discharge to evaluate neurological function.

\section{Radiological Assessment and DTI/DTT Reconstruction}

All patients underwent MRI examinations using the Magnetom TIM Trio 3.0-T MRI machine (Siemens Healthcare) with the same parameters. DTI images were collected using the diffusion-weighted echo-planar imaging technique with the following parameters: diffusion weighting coefficient $1000 \mathrm{sec} / \mathrm{mm}^{2}, 26$ orientations, TR $4600 \mathrm{msec}$, TE $82.90 \mathrm{msec}$, slice thickness $4.0 \mathrm{~mm}, 40$ slices, and display field of view (DFOV) $240 \mathrm{~mm}$. Additionally, a 3D T1weighted anatomical sequence was also collected alongside the DTI scan using a gradient echo sequence with the following parameters: TR $8.21 \mathrm{msec}$, TE $3.22 \mathrm{msec}$, slice thickness $1.20 \mathrm{~mm}$, 176 slices, and DFOV $240 \mathrm{~mm}$.

DTI data processing was performed on a StealthViz Planning Station (Medtronic). A fractional anisotropy threshold of 0.10 , minimum fiber length of $40 \mathrm{~mm}$, and maximum turning angle of $45^{\circ}$ were used for DTT reconstruction. By following a region of interest (ROI) approach, the bilateral CST and medial lemniscus (ML) were reconstructed and demonstrated, although only CSTs were evaluated in this study. The posterior limb of the inner capsule, pyramids of the medulla oblongata, pedunculus cerebri, and front parts of the pons were usually chosen as the ROIs. Two cases showing the reconstruction results of CSTs are illustrated in Fig. 1.

The DTI/DTT results were evaluated by two neurosurgeons. The CST status was then classified into four grades as previously reported by Lazar et al.: 1) normal, 2) deviated, 3) deformed, and 4) interrupted. ${ }^{14}$ Each CST was scored as $0,2,3$, or 4 based on Lazar et al.'s grading system. ${ }^{14}$ In addition, the tumor-to-CST distance (TCD) was measured and defined by the closest distance from the tumor to the CST on axial T1-weighted MR images.

\section{Surgical Protocol}

The surgical approach was selected individually depending on the characteristics of lesions on MRI (e.g., location, size, growth pattern), a thorough assessment of preoperative neurofunction, and the DTI/DTT findings. For each patient, approach selections were routinely performed twice. The first selection was performed without DTI/DTT results. Then, DTI/DTT was taken into consideration, and the approach was selected again by the same operator. Both selections were recorded in the medical documents, making this retrospective investigation possible.

For the safety of the surgery and to improve the extent of resection as much as possible, neuronavigation and electrophysiological monitoring were routinely used. The contour of the tumor and spatial correlation of the tumor to CST and ML could be visualized on the same image of the StealthStation Treon Plus (Medtronic) or StealthStation S7 surgical navigation system (Medtronic). If a decrease in the amplitude of motor evoked potentials (MEPs) or a repeated abnormal alert of free-running electromyography was noted during surgery, the surgeon would stop manipulation, irrigate the operating field with warm saline, check the actual position with navigation, 

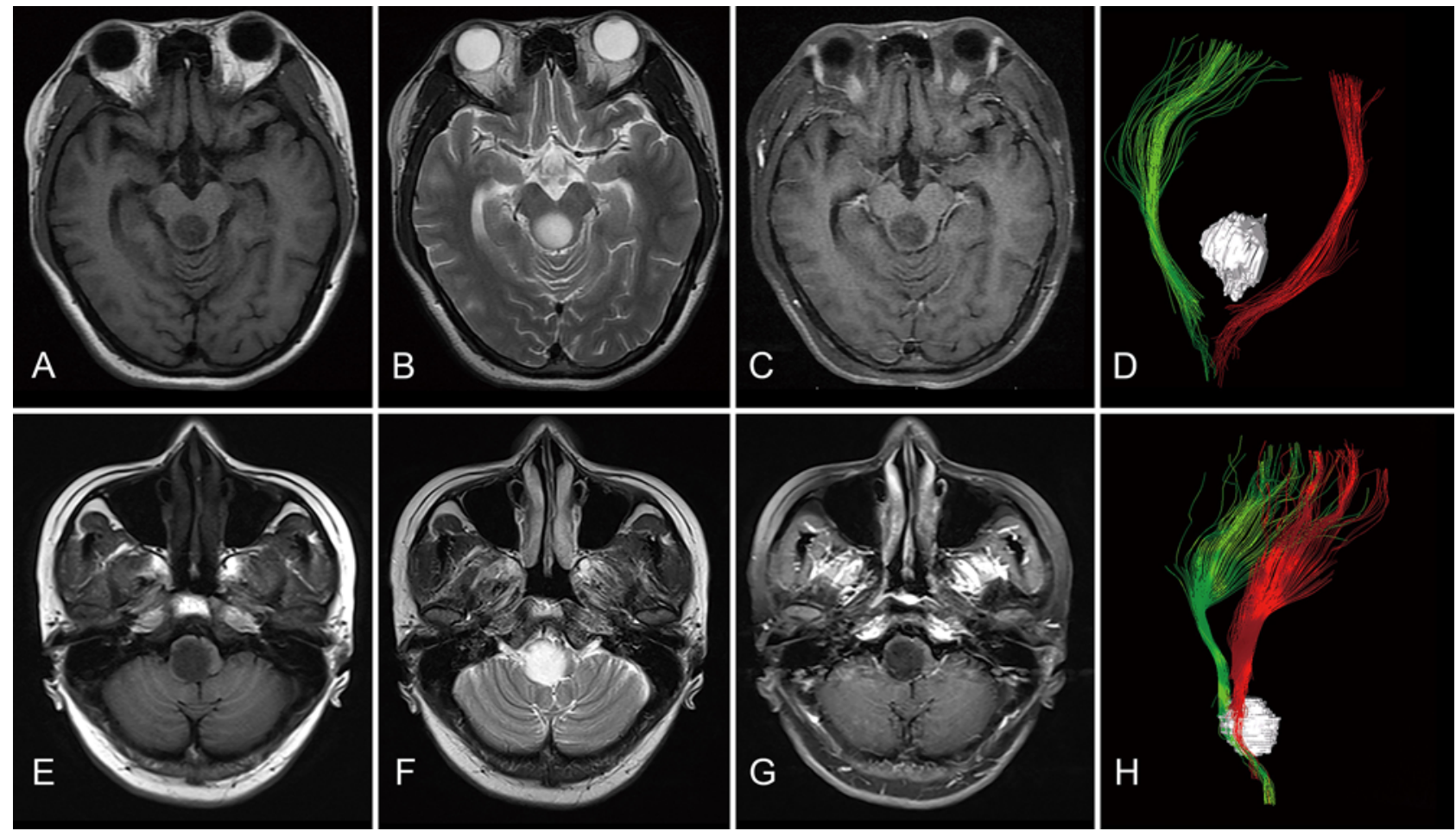

FIG. 1. Cases of reconstruction of the CST using DTI data. A-D: A case of a midbrain glioma. The lesion showed high signals in T2-weighted MR images and low signals without enhancement in T1-weighted MR images. The bilateral CSTs (red and green) were normal. E-H: A case of a glioma in the medulla. The lesion showed high signals in T2-weighted MR images and low signals without enhancement in T1-weighted MR images. The left CST (red) was deviated by the lesion, while the right CST (green) was deformed.

and evaluate the extent of resection and distance to white matter tracts before continuing the surgery.

Regarding the brain shift and possible inaccuracy of DTI/DTT reconstruction, neuronavigation-guided DsCS was used to verify the authentic position of the CST during surgery, as we have previously reported. ${ }^{15}$ Specifically, the monopolar stimulator was connected to the SureTrak II adaptor (Medtronic); thus, it could function as both a navigation probe and electrical stimulator simultaneously. The validation procedure was performed after tumor removal but could be repeated at any stage of surgery when the tumor-brainstem interface was difficult to define. The intensity of DsCS was initially set at $5 \mathrm{~mA}$ and could be increased to a maximum of $20 \mathrm{~mA}$. A positive DsCS was confirmed and documented by MEP changes in the hand or foot within a distance of $2 \mathrm{~mm}$ from the stimulator to the position of the reconstructed CST displayed by neuronavigation. A negative DsCS was recorded if at least three stimulations failed to elicit a potential with fine adjustment of the location of the probe head or augmentation of intensity; related cases are shown in Fig. 2.

\section{Statistical Analysis}

Statistical analyses were performed using IBM SPSS software (version 23.0, IBM Corp.). For measurement data, if the variables obeyed a normal distribution, means and standard deviations were calculated; t-tests or ANOVAs were used for intergroup comparisons. Otherwise, quar- tiles were calculated, and the Mann-Whitney U-test or Kruskal-Wallis H-test was used for intergroup comparisons. For categorical data, frequencies and percentages were calculated, and chi-square tests were used for intergroup comparisons. The Spearman correlation was used to analyze the correlation between quantitative parameters of DTI/DTT, including the CST score and TCD, and muscle strength and mRS score. Multiple linear regressions were used to analyze the predictive value of DTI/ DTT parameters for the postoperative muscle strength and mRS score. A $p$ value $<0.05$ was considered statistically significant. Concerning the mRS analyses, the CST score of each patient was defined as the higher value of the bilateral CST scores, while the TCD was defined as the lower value of the bilateral TCDs.

\section{Results}

\section{Patient Characteristics and Radiological Assessments}

A total of 54 patients were included in this study (Table 1). Among all patients, $23(42.6 \%)$ were female and 31 $(57.4 \%)$ were male. The median age of the patients was 17.6 years (range 1.9-62.2 years). The duration of symptoms ranged from 0.7 to 240 months, with a median of 5.5 months. At admission, the frequency and percentage of preoperative mRS scores 1,2,3, and 4 were $34(63.0 \%), 13$ (24.1\%), 6 (11.1\%), and 1 (1.9\%), respectively. At discharge, the mRS scores of the included patients, with a median of 

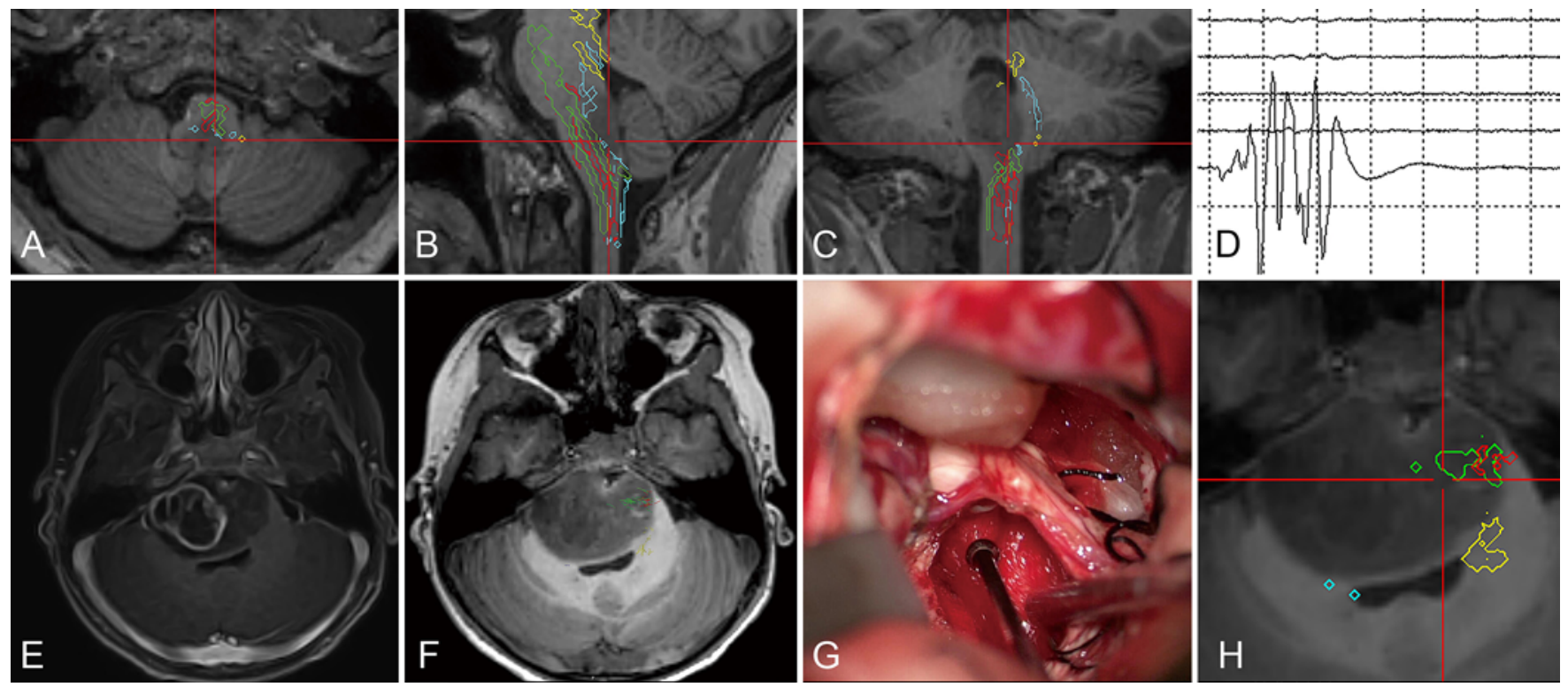

FIG. 2. Cases of spatial verification of reconstructed CSTs and brainstem glioma resection/cytoreduction performed with the assistance of DTI/DTT-merged neuronavigation and DsCS. A-D: Spatial verification of reconstructed CSTs. The pointing position (centers of red crosses) of the monopolar stimulator was confirmed by neuronavigation to indicate the inferior and ventral border of the tumor, which was close to the reconstructed bilateral CSTs (A-C). A positive DsCS was confirmed by MEP (D). Thus, the spatial accuracy of the reconstructed CSTs was validated. E-H: Assistance of DTI/DTT-merged neuronavigation and DsCS during brainstem glioma cytoreduction. A lesion with a significant enhancement area is shown on MRI (E). The bilateral CSTs were reconstructed using DTI images and found to be pushed to the left and enwrapped by the tumor (panel F: green, right CST; red, left CST; blue, right ML; yellow, left ML). Thus, it was critical to determine the accurate location of the CSTs and boundary of tumor cytoreduction. During the operation, navigation combined with DsCS was repeated to confirm the spatial location of surgical manipulation and protect the fibers from injury (G). With the assistance of DTI-merged navigation and DsCS, the boundary of tumor cytoreduction was set near the displayed position (center of red cross), which was confirmed twice using both techniques $(\mathrm{H})$. Consequently, a balance between the extent of resection and protection of neurofunctions was achieved.

3, were higher than at admission. A detailed follow-up and survival analysis were beyond the scope of this study.

In this study, 31 tumors $(57.4 \%)$ were located in the medulla, $10(18.5 \%)$ in the pontine, and the others (13, $24.1 \%$ ) in the midbrain. The mean maximum diameter of the lesions on axial T2-weighted MRI was $24.6 \pm 8.1 \mathrm{~mm}$. Thirty tumors (55.6\%) were intrinsic, whereas 24 (44.4\%) were exophytic. Most tumors $(39,72.2 \%)$ presented with ring or irregular enhancement, while 15 lesions (27.8\%) did not exhibit enhancement after the injection of contrast. Six patients had normal bilateral CSTs, 12 patients had unilateral CST impairments, and 36 patients had bilateral CSTs involved. The most common changes in CSTs were deformation $(n=29)$, followed by deviation $(n=28)$ and interruption $(n=27)$. As the TCD did not follow a normal distribution, the quartiles were $0.00 \mathrm{~mm}, 0.00 \mathrm{~mm}$, and $3.10 \mathrm{~mm}$. A summary of the patient information, including demographic and symptomatic data, is provided in Table 1.

\section{Influence of DTI/DTT Findings on Surgical Approach Selection and Intraoperative Validation of the Spatial Accuracy of the Reconstructed CST}

Without the assistance of DTI/DTT findings, the far lateral approach $(22,40.7 \%)$ was most commonly selected, followed by the midline suboccipital approach $(13,24.1 \%)$ and the suboccipital transtentorial approach (11, 20.4\%; Table 2). The rest of the approaches consisted of the sub- temporal $(5,9.3 \%)$, retrosigmoid $(2,3.7 \%)$, and temporaloccipital approaches $(1,1.9 \%)$.

After taking the DTI/DTT findings into consideration, the most commonly used approach was the midline suboccipital approach $(23,42.6 \%)$, and the rest consisted of the suboccipital transtentorial $(11,20.4 \%)$, far lateral (11, $20.4 \%)$, subtemporal $(4,7.4 \%)$, retrosigmoid $(3,5.6 \%)$, and temporal-occipital approaches $(2,3.7 \%)$. In total, 18 patients (33.3\%) experienced a modification of surgical approaches. The changes in surgical approaches are shown in Table 2.

Among 108 reconstructed CSTs of 54 included patients, 16 CSTs presented with severe hemiparesis of related limbs (muscle strength $\leq 3$ ). In this context, the transcranial MEPs could not be elicited, nor could DsCS be conducted. DsCS of 37 CSTs could not be performed, as they were far away from the tumor border. For the other 55 CSTs, positive results of DsCS were recorded in 46 cases $(83.6 \%)$ when placing the stimulator within the $2-\mathrm{mm}$ range of the imaged CST.

\section{Relationship Between DTI/DTT Findings of CSTs and Muscle Strength}

A cross table was drawn concerning the pre- and postoperative muscle strength of the related limbs of 108 CSTs in 54 patients (Table 3 ). Preoperative muscle strength positively correlated with CST score $(r=0.358, p<0.001)$. The 
TABLE 1. Summary of clinical data

\begin{tabular}{|c|c|c|}
\hline Variable & Value & Range (IQR) \\
\hline No. of patients & 54 & \\
\hline Males/females (\%) & $31(57.4) / 23(42.6)$ & \\
\hline Median age, yrs & 17.6 & $1.9-62.2(8.8,36.2)$ \\
\hline $\begin{array}{l}\text { Median duration of } \\
\text { symptoms, mos }\end{array}$ & 5.5 & $0.7-240.0(2.0,19.5)$ \\
\hline \multicolumn{3}{|l|}{ Preop symptoms, n (\%) } \\
\hline Weaker muscle strength & $18(33.3)$ & \\
\hline $\begin{array}{l}\text { Lower cranial nerve } \\
\text { deficits }\end{array}$ & $26(48.1)$ & \\
\hline Hearing loss & $7(13.0)$ & \\
\hline Facial paralysis & $7(13.0)$ & \\
\hline $\begin{array}{l}\text { Trigeminal nerve } \\
\text { dysfunction }\end{array}$ & $13(24.1)$ & \\
\hline $\begin{array}{l}\text { Extraocular muscle } \\
\text { dysfunction }\end{array}$ & $16(29.6)$ & \\
\hline Headache or dizziness & $28(51.9)$ & \\
\hline Median preop mRS score & 1 & $1-4(1,2)$ \\
\hline \multicolumn{3}{|l|}{ Lesion position, $\mathrm{n}(\%)$} \\
\hline Midbrain & $13(24.1)$ & \\
\hline Pons & $10(18.5)$ & \\
\hline Medulla & $31(57.4)$ & \\
\hline $\begin{array}{l}\text { Mean max diameter of } \\
\text { lesion } \pm \mathrm{SD}, \mathrm{mm}\end{array}$ & $24.6 \pm 8.1$ & \\
\hline \multicolumn{3}{|l|}{ Growth pattern, n (\%) } \\
\hline Intrinsic & $30(55.6)$ & \\
\hline Exophytic & $24(44.4)$ & \\
\hline \multicolumn{3}{|l|}{ CST classification, $n(\%)^{*}$} \\
\hline Normal & $24(22.2)$ & \\
\hline Deviated & $28(25.9)$ & \\
\hline Deformed & $29(26.9)$ & \\
\hline Interrupted & $27(25.0)$ & \\
\hline \multicolumn{3}{|l|}{ Enhancement of lesion, $n(\%)$} \\
\hline With enhancement & $39(72.2)$ & \\
\hline Without enhancement & $15(27.8)$ & \\
\hline Median TCD, mm & 0.00 & $0.0-14.6(0.00,3.10)$ \\
\hline Median postop mRS score & 3 & $1-5(2,4)$ \\
\hline \multicolumn{3}{|l|}{$\begin{array}{l}\text { Surgery-associated } \\
\text { complications, } \mathrm{n}(\%)\end{array}$} \\
\hline $\begin{array}{l}\text { Deterioration of } \\
\text { neurological deficit }\end{array}$ & $7(13.0)$ & \\
\hline $\begin{array}{l}\text { New developed } \\
\text { neurological symptoms }\end{array}$ & $29(53.7)$ & \\
\hline General complications, n (\%) & $19(35.2)$ & \\
\hline Hydrocephalus & $3(5.6)$ & \\
\hline Pneumonia & $11(20.4)$ & \\
\hline Surgical site infection & $5(9.3)$ & \\
\hline \multicolumn{3}{|l|}{ Pathological results, $n(\%)$} \\
\hline WHO grade I & $12(22.2)$ & \\
\hline Ganglioglioma & $8(14.8)$ & \\
\hline Pilocytic astrocytoma & $4(7.4)$ & \\
\hline
\end{tabular}

CONTINUED IN NEXT COLUMN »
» CONTINUED FROM PREVIOUS COLUMN

TABLE 1. Summary of clinical data

\begin{tabular}{lcc}
\hline \multicolumn{1}{c}{ Variable } & Value & Range (IQR) \\
\hline $\begin{array}{l}\text { Pathological results, } \mathrm{n}(\%) \\
\text { (continued) }\end{array}$ & \\
\hline WHO grade II & $27(50.0)$ \\
\hline Astrocytoma & $19(35.2)$ \\
\hline Oligodendroglioma & $3(5.6)$ \\
\hline Oligoastrocytoma & $5(9.3)$ \\
\hline WHO grade III & $10(18.5)$ \\
\hline Anaplasia astrocytoma & $7(13.0)$ \\
\hline $\begin{array}{l}\text { Anaplasia } \\
\text { oligodendroglioma }\end{array}$ & $1(1.9)$ \\
\hline $\begin{array}{l}\text { Anaplasia } \\
\text { oligoastrocytoma }\end{array}$ & $2(3.7)$ \\
\hline WHO grade IV & $5(9.3)$ \\
\hline Glioblastoma & $5(9.3)$ \\
\hline
\end{tabular}

* There were 108 total CSTs.

CST scores did not share the same postoperative muscle strength distribution, as indicated by the Kruskal-Wallis H-test $\left(\chi^{2}=18.715, p<0.001\right)$. A Spearman correlation analysis indicated that CST score was negatively related to postoperative muscle strength $(\mathrm{r}=-0.395, \mathrm{p}<0.001)$. Moreover, the TCD was positively related to postoperative muscle strength $(r=0.275, p=0.004)$. When univariate linear regression was performed, gender, preoperative muscle strength, maximum lesion diameter, and CST score were linearly associated with postoperative muscle strength. However, only gender $(\mathrm{t}=2.346, \mathrm{p}=0.021)$ and CST score $(\mathrm{t}=-2.461, \mathrm{p}=0.016)$ had an independent linear association with postoperative muscle strength on multivariate analysis (Table 4).

\section{Relationship Between DTI/DTT Findings of CSTs and Postoperative mRS Scores}

The CST scores did not share the same postoperative mRS score distribution as indicated by the Kruskal-Wallis H-test $\left(\chi^{2}=12.394, p=0.006\right)$. CST score was positively related to postoperative $\mathrm{mRS}$ score $(\mathrm{r}=0.430, \mathrm{p}=0.001)$, while TCD was negatively related to postoperative mRS score $(r=-0.329, p=0.015)$. When univariate linear regression was performed, preoperative muscle strength, preoperative mRS score, and CST score were linearly associated with postoperative mRS score. In addition, preoperative $\mathrm{mRS}$ score $(\mathrm{t}=2.532, \mathrm{p}=0.015)$ and CST score $(\mathrm{t}=2.052, \mathrm{p}=0.046)$ had an independent linear association with postoperative $\mathrm{mRS}$ score on multivariate analysis (Table 5).

\section{Discussion}

Since the advent of DTI/DTT technology, it has been applied to the surgical planning of patients with supratentorial gliomas. Studies have shown that DTI/DTT can not only improve the extent of tumor resection, but also reduce the risk of postoperative neurological deficits and conse- 
TABLE 2. Surgical approach selection before and after considering DTI results

\begin{tabular}{|c|c|c|c|c|c|c|c|}
\hline \multirow[b]{2}{*}{$\begin{array}{c}\text { Approach After } \\
\text { Considering DTI Results }\end{array}$} & \multicolumn{6}{|c|}{ Approach Before Considering DTI Results } & \multirow[b]{2}{*}{ Tota } \\
\hline & $\begin{array}{l}\text { Suboccipital } \\
\text { Midline }\end{array}$ & $\begin{array}{c}\text { Far } \\
\text { Lateral }\end{array}$ & Retrosigmoid & Subtemporal & $\begin{array}{l}\text { Suboccipital } \\
\text { Transtentorial }\end{array}$ & Temporal-Occipital & \\
\hline Suboccipital midline & 12 & 11 & 0 & 0 & 0 & 0 & 23 \\
\hline Far lateral & 1 & 10 & 0 & 0 & 0 & 0 & 11 \\
\hline Retrosigmoid & 0 & 1 & 1 & 1 & 0 & 0 & 3 \\
\hline Subtemporal & 0 & 0 & 1 & 2 & 0 & 1 & 4 \\
\hline Suboccipital transtentorial & 0 & 0 & 0 & 0 & 11 & 0 & 11 \\
\hline Temporal-occipital & 0 & 0 & 0 & 2 & 0 & 0 & 2 \\
\hline Total & 13 & 22 & 2 & 5 & 11 & 1 & 54 \\
\hline
\end{tabular}

quently improve the long-term quality of life of patients..$^{13,16}$ In recent years, a few studies have focused on the application of DTI/DTT in the surgical management of brainstem lesions, the majority of which were brainstem cavernous malformations. ${ }^{9-12,17-21}$ DTI/DTT was found to be helpful in surgical planning, including optimal approaches and safe entry zones. Additionally, it may have promising value for the prediction of postoperative neurological function..$^{12,15,19}$ However, as brainstem gliomas behave differently from cavernous malformations and their ability to infiltrate the normal brain may vary depending on their genetic characteristics, the role of DTI/DTT in brainstem glioma surgery merits investigation. In our study, the number of changes in the CSTs in the deviation, deformation, and interruption groups were very similar. In contrast, white matter tracts are more frequently deviated rather than destroyed by the lesions in brainstem cavernous malformations. ${ }^{10,12}$ This discrepancy could be explained by the invasive nature of gliomas and indicate that these critical fibers are more vulnerable to surgical manipulation during an operation, thus meriting significant protective efforts.

Understanding the anatomical relationship between the tumor and tracts is helpful to determine the approach, and many studies have shown that DTI/DTT is a highly valuable tool in preoperative surgical planning., ${ }^{9} 10,12,19$ In our series, more than one-third of the approaches were adjusted after taking the DTI/DTT results into account. The main reason for this adjustment was that the white matter tracts were displaced more superficially by the tumor in the surgical corridor, which prevented adequate exposure and safe resection. The changes were mostly from the far lateral approach to the suboccipital midline approach. Most of these cases had lesions located in the ventral or ventrolateral medulla. The tumors expanded from the front or lateral part of the medulla, or surrounding tissue was thin there. However, results of DTI/DTT suggested that CSTs were pushed forward or laterally by the tumor and very likely to appear in the surgical path. Because brainstem gliomas are intraaxial lesions and likely to be enwrapped by brainstem tissues, DTI/DTT is valuable for optimizing surgical strategies by indicating whether these tissues contain important fibers. As conventional MRI cannot predict the location of white matter tracts, DTI/DTT should be routinely performed in the resection of brainstem lesions but may also play an important role in stereotactic biopsy.
The accuracy of DTI/DTT changes has been studied since the emergence of this technique, especially when used in image-guided neuronavigation. The disadvantages inherent in image processing, such as artifacts, resolution, and parameters, may affect the validity of the reconstructed results. Additionally, the registration errors of the navigation system and the intraoperative brain shift effect may also lead to inaccurate estimation of white matter tracts when using DTI-merged neuronavigation. ${ }^{22}$ According to a review focused on the brain shift in neuronavigation, the mean displacement of the brain surface could be as great as $10 \mathrm{~mm} .{ }^{23}$ In this context, validation of the spatial accuracy of both the neuronavigation and reconstructed CSTs should be performed.

To validate neuronavigation, verifications of the neuronavigation-guided position of anatomical landmarks were periodically performed, especially before and during tumor resection. This verification procedure is shown in Fig. 3. Although no quantified measurements of the displacement of these landmarks were performed, we found that brain shifting of the brainstem during neuronavigation was almost imperceptible by nonmeasurement verification. We speculated that the surrounding structures, such as the cranial nerves, thalamus, and spinal cord, play a role in fixation of the brainstem, and the procedure of lesion exposure, which does not involve traction of the brainstem, contributes to a higher accuracy of neuronavigation in brainstem surgeries than in supratentorial surgeries. However, the reason or mechanism clearly requires further investigation.

For validation of reconstructed CSTs, direct cortical stimulation and DsCS are considered the gold standard for verifying the accuracy of tractography in the surgical treatment of supratentorial gliomas in the eloquent area. ${ }^{24}$ Good correspondence has been reported between DTI reconstructed tracts and positive electrical stimulation sites, ranging from $92 \%$ to $95 \% .{ }^{25-27}$ However, these results were limited to the spectrum of supratentorial lesions. Validation of tractography has never been investigated in brainstem surgery. In the present study, we found that the efficacy and accuracy of DTI/DTT were comparable to those in previous reports. Although DsCS results may be affected by many factors, including the stimulation parameters, anesthetic regimens, electric current spread, and resistance, ${ }^{27-29}$ the high positive rate of intraopera- 
TABLE 3. Cross table on CST classification and muscle strength of related limbs

\begin{tabular}{lrlllllr}
\hline \multirow{2}{*}{$\begin{array}{c}\text { CST } \\
\text { Classification }\end{array}$} & 0 & I & II & III & IV & V & Total \\
\cline { 2 - 6 } Preop & & & & & & & \\
\hline Normal & 0 & 0 & 0 & 0 & 0 & 24 & 24 \\
\hline Deviated & 0 & 0 & 0 & 0 & 6 & 22 & 28 \\
\hline Deformed & 0 & 0 & 1 & 3 & 6 & 19 & 29 \\
\hline Interrupted & 0 & 0 & 0 & 1 & 11 & 15 & 27 \\
\hline Total & 0 & 0 & 1 & 4 & 23 & 80 & 108 \\
\hline Postop & & & & & & & \\
\hline Normal & 0 & 0 & 0 & 3 & 3 & 18 & 24 \\
\hline Deviated & 0 & 0 & 0 & 0 & 8 & 20 & 28 \\
\hline Deformed & 1 & 1 & 1 & 2 & 11 & 13 & 29 \\
\hline Interrupted & 2 & 1 & 1 & 4 & 12 & 7 & 27 \\
\hline Total & 3 & 2 & 2 & 9 & 34 & 58 & 108 \\
\hline
\end{tabular}

tive DsCS in our study indicated that the reconstructed CST corresponded well to its real location with current techniques. Our study suggested that DTT-merged neuronavigation could be comfortably utilized as a reliable tool in surgery for brainstem gliomas. However, a mismatch between tractography and DsCS was identified. To further determine the contributing factors of the mismatch, a study that quantifies the brain shift effect on the brainstem and measures the distance of stimulation sites in relation to the tumor and reconstructed CST is currently in progress.

The working mechanisms of DTI-merged neuronavigation and DsCS make them a naturally complementary pair, as one modality focuses on imaging-based anatomical positioning and the other on real-time functional positioning. ${ }^{23,30,31}$ During surgery, they can corroborate each other, and thus the position of CSTs may be validated at both levels. Furthermore, if the brain shift is significant and neuronavigation becomes less reliable, or in contrast, DsCS is not available because of severe preoperative damage to fiber tracts by the tumor, administration of muscle relaxants, and device failure (among others), the other modality can assume the tasks, helping to provide alerts about interferences with tracts of interest by surgical manipulation. Given these considerations, it is advisable to combine these two modalities, and other techniques such as intraoperative electrophysical monitoring (transcranial MEPs and somatosensory evoked potentials) are also recommended when assessing the extent of resection and preservation of function.

A doubtful virtue of DTI/DTT is the predictive value for postoperative neurological function. By qualitatively and quantitively evaluating the DTI/DTT results, Lin et al. found that CST score and lesion-to-CST distance were correlated with short-term postoperative neurological function, whereas others did not find such a correlation of DTI/DTT changes to mRS score at follow-up. ${ }^{10-12,19,21}$ In comparison, we found that the CST score was an independent predictor of postoperative muscle strength and mRS score at discharge. As CST scores reflect the severity of
TABLE 4. Relationship between postoperative muscle strength and preoperative variables shown by linear regression

\begin{tabular}{|c|c|c|c|c|}
\hline \multirow[b]{2}{*}{ Variable } & \multicolumn{2}{|c|}{$\begin{array}{c}\text { Univariate } \\
\text { Analysis }\end{array}$} & \multicolumn{2}{|c|}{$\begin{array}{c}\text { Multivariate } \\
\text { Analysis }\end{array}$} \\
\hline & $t$ & $p$ Value & $t$ & $p$ Value \\
\hline Age & 0.227 & 0.821 & -0.512 & 0.610 \\
\hline Gender & 2.211 & 0.029 & 2.346 & 0.021 \\
\hline Preop duration & 0.974 & 0.346 & & \\
\hline Preop muscle strength & 2.001 & 0.048 & 1.150 & 0.253 \\
\hline Preop mRS score & 0.448 & 0.655 & & \\
\hline Location of lesion & -1.336 & 0.184 & & \\
\hline Max lesion diameter & -2.039 & 0.044 & -1.037 & 0.302 \\
\hline Growth pattern of lesion & 0.172 & 0.864 & & \\
\hline Enhancement of lesion & 1.877 & 0.063 & & \\
\hline CST score & -3.587 & 0.001 & -2.461 & 0.016 \\
\hline TCD & 1.588 & 0.115 & & \\
\hline
\end{tabular}

Boldface type indicates statistical significance.

injury to the pyramidal tracts, a high score indicates that the tracts have already been damaged and are more vulnerable to surgical manipulation; in this case, patients are very likely to suffer from deteriorated or new neurological deficits, thus correlating with weaker muscle strength and worse postoperative mRS score. Additionally, and in contrast to previous reports, we noted that TCD was not associated with the postoperative mRS score. Unlike the anatomical relationship between the CST and brainstem cavernous malformation, most CSTs were populated close to the tumor in patients with brainstem gliomas, and thus TCD loses its predictive value.

Although DTI/DTT has been developed with increasing robustness, it still has some limitations. Leclercq et al. have summarized the factors that may affect the validation of DTI/DTT as follows: crossing fibers, spurious fibers, magnetic susceptibility artifacts, tumor infiltration and tu-

TABLE 5. Relationship between postoperative mRS score and preoperative variables shown by linear regression

\begin{tabular}{|c|c|c|c|c|}
\hline \multirow[b]{2}{*}{ Variable } & \multicolumn{2}{|c|}{$\begin{array}{c}\text { Univariate } \\
\text { Analysis }\end{array}$} & \multicolumn{2}{|c|}{$\begin{array}{c}\text { Multivariate } \\
\text { Analysis }\end{array}$} \\
\hline & $\mathrm{t}$ & $p$ Value & $t$ & $p$ Value \\
\hline Age & -0.270 & 0.788 & -0.236 & 0.815 \\
\hline Gender & -0.893 & 0.376 & -1.234 & 0.223 \\
\hline Preop duration & -0.507 & 0.615 & & \\
\hline Preop muscle strength & -2.775 & 0.008 & -0.157 & 0.876 \\
\hline Preop mRS score & 3.642 & 0.001 & 2.532 & 0.015 \\
\hline Location of lesion & 2.084 & 0.042 & & \\
\hline Max lesion diameter & 1.068 & 0.291 & & \\
\hline Growth pattern of lesion & 1.305 & 0.198 & & \\
\hline Enhancement of lesion & -1.213 & 0.231 & & \\
\hline CST score & 3.160 & 0.003 & 2.052 & 0.046 \\
\hline TCD & -1.711 & 0.093 & & \\
\hline
\end{tabular}

Boldface type indicates statistical significance. 

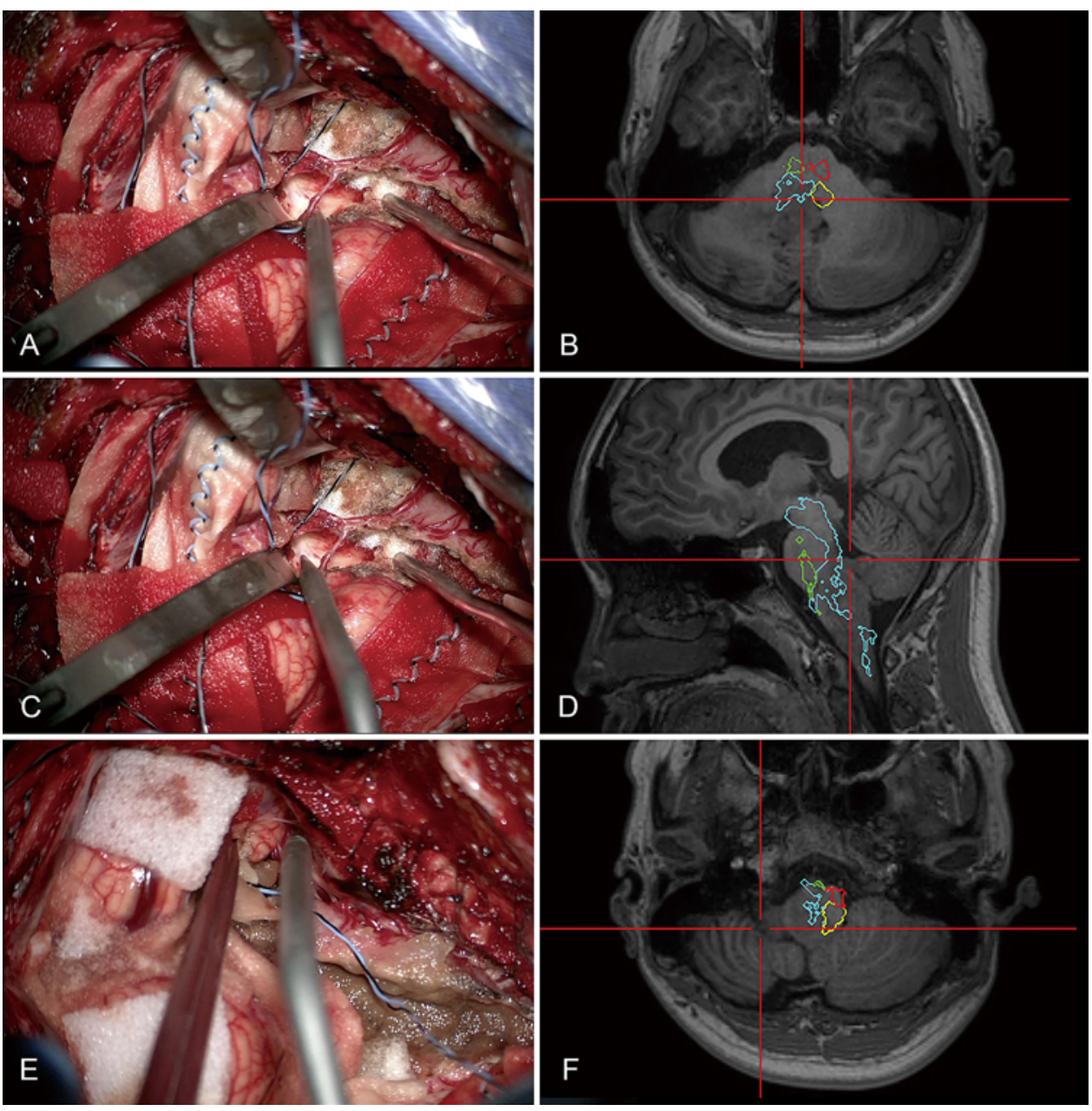

FIG. 3. A case of intraoperative verification of the neuronavigation spatial accuracy. To prevent the decrease in neuronavigation accuracy caused by brain shifting, verifications were periodically and repeatedly performed by pointing the probe to known anatomical landmarks. During resection of a tumor located in the medulla and cervical cord, the postmidline of the medulla ( $A$ and $\mathbf{B})$, upper border (C and D), and lateral border ( $\mathbf{E}$ and $\mathbf{F})$ of the resected tumor were used as landmarks. The centers of the red crosses point to the position of the probe recognized by neuronavigation.

mor mass effect, selection of ROIs, reconstruction parameters, brain shifting, and coregistration pitfalls. ${ }^{32}$ These factors may contribute to the high false positivity of DTI/ DTT results despite their excellent sensitivity and negative predictive values. ${ }^{21}$ Therefore, Duffau doubted the efficacy of DTI/DTT and considered it a research rather than a clinical tool. ${ }^{33}$ However, we do not share such an opinion because our results demonstrated a good accuracy of tractography, and we are comfortable using DTI/DTT routinely. Each radiological imaging modality has pitfalls, and it is the surgeon's responsibility to interpret these results correctly. In addition, recently developed techniques such as high angular resolution diffusion imaging (HARDI), generalized q-sampling imaging (GQI), and high-definition fiber tractography (HDFT) have demonstrated promising value to overcome the shortcomings of DTI/DTT and provide more detailed and accurate information about white matter tracts. ${ }^{8,24,34,35}$ These techniques, however, are mostly used for preoperative assessment rather than intraoperative real-time imaging guidance.
To the best of our knowledge, this study included the largest series investigating the utility of DTI/DTT in brainstem glioma surgery. Yet, the present investigation has the following limitations. First, its retrospective nature and single-arm design may have biased the interpretation of our results. Second, bilateral trigeminal lemniscus and visual and auditory conduction pathways were not reconstructed and studied. A better-designed prospective study will be conducted to further justify the use of DTI/DTT in brainstem glioma surgery. Nevertheless, the results of our study suggest that DTI/DTT is a valuable tool for evaluating brainstem lesions and guiding resection, and it may be routinely used together with other multimodal techniques when a surgical decision is made and surgery is being performed.

\section{Conclusions}

DTI/DTT is a valuable tool in the surgical management of brainstem gliomas. With good spatial accuracy, it can 
help optimize surgical planning, guide tumor resection, and predict postoperative muscle strength and quality of life.

\section{Acknowledgments}

This study was funded by the Capital Characteristic Clinical Application Project (no. Z181100001718196). We thank all the colleagues who participated in the surgical treatment of brainstem gliomas in our institute but did not fulfill the criteria for authorship.

\section{References}

1. Guillamo JS, Monjour A, Taillandier L, et al. Brainstem gliomas in adults: prognostic factors and classification. Brain. 2001;124(pt 12):2528-2539.

2. Jallo GI, Biser-Rohrbaugh A, Freed D. Brainstem gliomas. Childs Nerv Syst. 2004;20(3):143-153.

3. Smith MA, Freidlin B, Ries LA, Simon R. Trends in reported incidence of primary malignant brain tumors in children in the United States. J Natl Cancer Inst. 1998;90(17):1269-1277.

4. Choux M, Di Rocco C, Hockley AD, Walker ML. Pediatric Neurosurgery. Churchill Livingstone; 1999.

5. Klimo P Jr, Pai Panandiker AS, Thompson CJ, et al. Management and outcome of focal low-grade brainstem tumors in pediatric patients: the St. Jude experience. J Neurosurg Pediatr. 2013;11(3):274-281.

6. Sinha S, Kale SS, Chandra SP, et al. Brainstem gliomas: surgical indications and technical considerations in a series of 58 cases. Br J Neurosurg. 2014;28(2):220-225.

7. Teo C, Siu TL. Radical resection of focal brainstem gliomas: is it worth doing? Childs Nerv Syst. 2008;24(11):1307-1314.

8. Berman J. Diffusion MR tractography as a tool for surgical planning. Magn Reson Imaging Clin N Am. 2009;17(2): 205-214.

9. Chen X, Weigel D, Ganslandt O, et al. Diffusion tensor imaging and white matter tractography in patients with brainstem lesions. Acta Neurochir (Wien). 2007;149(11):1117-1131.

10. Flores BC, Whittemore AR, Samson DS, Barnett SL. The utility of preoperative diffusion tensor imaging in the surgical management of brainstem cavernous malformations. $J$ Neurosurg. 2015;122(3):653-662.

11. Kovanlikaya I, Firat Z, Kovanlikaya A, et al. Assessment of the corticospinal tract alterations before and after resection of brainstem lesions using diffusion tensor imaging (DTI) and tractography at 3T. Eur J Radiol. 2011;77(3):383-391.

12. Li D, Jiao YM, Wang L, et al. Surgical outcome of motor deficits and neurological status in brainstem cavernous malformations based on preoperative diffusion tensor imaging: a prospective randomized clinical trial. J Neurosurg. 2018; 130(1):286-301.

13. Wu JS, Zhou LF, Tang WJ, et al. Clinical evaluation and follow-up outcome of diffusion tensor imaging-based functional neuronavigation: a prospective, controlled study in patients with gliomas involving pyramidal tracts. Neurosurgery. 2007;61(5):935-949.

14. Lazar M, Alexander AL, Thottakara PJ, et al. White matter reorganization after surgical resection of brain tumors and vascular malformations. AJNR Am J Neuroradiol. 2006; 27(6):1258-1271.

15. Li Z, Wang M, Zhang L, et al. Neuronavigation-guided corticospinal tract mapping in brainstem tumor surgery: better preservation of motor function. World Neurosurg. 2018;116: e291-e297.

16. Essayed WI, Zhang F, Unadkat P, et al. White matter tractography for neurosurgical planning: a topography-based review of the current state of the art. Neuroimage Clin. 2017;15: 659-672.
17. Faraji AH, Abhinav K, Jarbo K, et al. Longitudinal evaluation of corticospinal tract in patients with resected brainstem cavernous malformations using high-definition fiber tractography and diffusion connectometry analysis: preliminary experience. J Neurosurg. 2015;123(5):1133-1144.

18. Januszewski J, Albert L, Black K, Dehdashti AR. The usefulness of diffusion tensor imaging and tractography in surgery of brainstem cavernous malformations. World Neurosurg. 2016;93:377-388.

19. Lin Y, Lin F, Kang D, et al. Supratentorial cavernous malformations adjacent to the corticospinal tract: surgical outcomes and predictive value of diffusion tensor imaging findings. $J$ Neurosurg. 2018;128(2):541-552.

20. Ulrich NH, Kockro RA, Bellut D, et al. Brainstem cavernoma surgery with the support of pre- and postoperative diffusion tensor imaging: initial experiences and clinical course of 23 patients. Neurosurg Rev. 2014;37(3):481-492.

21. Yao Y, Ulrich NH, Guggenberger R, et al. Quantification of corticospinal tracts with diffusion tensor imaging in brainstem surgery: prognostic value in 14 consecutive cases at 3T magnetic resonance imaging. World Neurosurg. 2015;83(6): 1006-1014.

22. Potgieser AR, Wagemakers M, van Hulzen AL, et al. The role of diffusion tensor imaging in brain tumor surgery: a review of the literature. Clin Neurol Neurosurg. 2014;124:51-58.

23. Gerard IJ, Kersten-Oertel M, Petrecca K, et al. Brain shift in neuronavigation of brain tumors: a review. Med Image Anal. 2017:35:403-420.

24. Celtikci P, Fernandes-Cabral DT, Yeh FC, et al. Generalized q-sampling imaging fiber tractography reveals displacement and infiltration of fiber tracts in low-grade gliomas. Neuroradiology. 2018;60(3):267-280.

25. Bello L, Gambini A, Castellano A, et al. Motor and language DTI Fiber Tracking combined with intraoperative subcortical mapping for surgical removal of gliomas. Neuroimage. 2008; 39(1):369-382.

26. Ohue S, Kohno S, Inoue A, et al. Accuracy of diffusion tensor magnetic resonance imaging-based tractography for surgery of gliomas near the pyramidal tract: a significant correlation between subcortical electrical stimulation and postoperative tractography. Neurosurgery. 2012;70(2):283-294.

27. Zhu FP, Wu JS, Song YY, et al. Clinical application of motor pathway mapping using diffusion tensor imaging tractography and intraoperative direct subcortical stimulation in cerebral glioma surgery: a prospective cohort study. Neurosurgery. 2012;71(6):1170-1184.

28. Keles GE, Lundin DA, Lamborn KR, et al. Intraoperative subcortical stimulation mapping for hemispherical perirolandic gliomas located within or adjacent to the descending motor pathways: evaluation of morbidity and assessment of functional outcome in 294 patients. J Neurosurg. 2004; 100(3):369-375

29. Mandonnet E, Winkler PA, Duffau H. Direct electrical stimulation as an input gate into brain functional networks: principles, advantages and limitations. Acta Neurochir (Wien). 2010;152(2):185-193.

30. Shiban E, Krieg SM, Haller B, et al. Intraoperative subcortical motor evoked potential stimulation: how close is the corticospinal tract? J Neurosurg. 2015;123(3):711-720.

31. Gomez-Tames J, Kutsuna T, Tamura M, et al. Intraoperative direct subcortical stimulation: comparison of monopolar and bipolar stimulation. Phys Med Biol. 2018;63(22):225013.

32. Leclercq D, Delmaire C, de Champfleur NM, et al. Diffusion tractography: methods, validation and applications in patients with neurosurgical lesions. Neurosurg Clin N Am. 2011;22(2): 253-268, ix.

33. Duffau H. Diffusion tensor imaging is a research and educational tool, but not yet a clinical tool. World Neurosurg. 2014; 82(1-2):e43-e45. 
34. Abhinav K, Yeh FC, Mansouri A, et al. High-definition fiber tractography for the evaluation of perilesional white matter tracts in high-grade glioma surgery. Neuro Oncol. 2015;17(9): 1199-1209.

35. Kuhnt D, Bauer MH, Egger J, et al. Fiber tractography based on diffusion tensor imaging compared with high-angularresolution diffusion imaging with compressed sensing: initial experience. Neurosurgery. 2013;72(suppl 1):165-175.

\section{Disclosures}

The authors report no conflict of interest concerning the materials or methods used in this study or the findings specified in this paper.

\section{Author Contributions}

Conception and design: L Zhang, Xiao, Pan. Acquisition of data: Xiao, Kong, P Zhang, Chen, Wang, Qiao. Analysis and interpretation of data: Xiao, Kong. Drafting the article: L Zhang, Xiao, Kong, Sun. Critically revising the article: all authors. Reviewed submitted version of manuscript: all authors. Approved the final version of the manuscript on behalf of all authors: L Zhang. Administrative/technical/material support: L Zhang, Wu, JT Zhang. Study supervision: L Zhang, Wu, JT Zhang.

\section{Supplemental Information}

Online-Only Content

Supplemental material is available online.

Supplemental Illustrative Cases. https://thejns.org/doi/suppl/ 10.3171/2020.10.FOCUS20166.

\section{Correspondence}

Liwei Zhang: Beijing Tiantan Hospital, Capital Medical University, Beijing, People’s Republic of China. zhangliweittyy@163.com. 